\title{
КИНЕТИКА ЗАСЕЛЕНИЯ ТРИПЛЕТНОГО СОСТОЯНИЯ ПРИМЕСНОЙ МОЛЕКУЛЫ
}

\section{1. Введение}

Многоатомным молекулам свойственно существование возбужденного триплетного электронного состояния $T_{1}$, лежащего ниже первого возбужденного синглетного $\left(S_{1}\right)$ уровня [ [ $\left.{ }^{1}\right]$. Благодаря такому расположению $T_{1}$-уровень может быть эффективно заселен путем интерконверсии с $S_{1}$-состояния, что делает его доступным для изучения различными спектроскопическими методами.

Интерес к изучению триплетных состояний значительно повысился в связи с обнаружением фотоиндуцированного сигнала ЭПР от триплетного состояния хлорофилла в фотосинтезирующих системах $\left.{ }^{2}\right]$. Роль триплетных состояний в фотосинтезе в настоящее время интенсивно изучается.

В магнитном поле триплетное состояние расщепляется на три компонента, различающиеся по проекциям спинового момента на направление поля. Однако уже в отсутствие магнитного поля имеется маленькое т. н. расщепление в нулевом поле, обусловленное спин-орбитальным взаимодействием. Для молекулы, внедренной в твердотельную матрицу, возможна вызванная спин-решеточным взаимодействием релаксация между спиновыми подуровнями триплета, скорость которой сильно зависит от температуры. С целью «вымораживания» спин-решеточной релаксации (СРР) часто используют температуры около $1 \mathrm{~K}$.

За последние годы для изучения триплетного состояния был предложен ряд новых методик, основанных на резонансном поглощении излучения в микроволновой области за счет переходов между подуровнями триплетного состояния (см. обзор $\left.\left[{ }^{3}\right]\right)$.

Известен способ изучения триплетного состояния, заключающийся в измерении временного хода опустошения синглетных уровней, детектированного по интенсивности флуоресценции $\left[{ }^{4,5}\right]$. Нами в $\left[{ }^{6,7}\right]$ было показано, как этот способ можно использовать в качестве самостоятельного метода (метода флуоресцентного детектирования триплета $(Ф Д Т))$ для систематического исследования триплетных состояний.

Хотя основные закономерности затухания и заселения метастабильного (триплетного) состояния были выведены еще в 1948 г. Б. Я. Свешниковым $\left[{ }^{8}\right]$, ряд аспектов не был им затронут из-за отсутствия соответствующего экспериментального материала*. С другой стороны, и более поздние трактовки не исчерпали всех аспектов проблемы.

* В частности, отсутствовали лазерные источники возбуждения и не применялись методы микроволновых резонансов. 
В работах по спиновой поляризации $\left[{ }^{9,10}\right]$ использовался вывод о том, что короткими световыми импульсами можно создавать неравновесные распределения между спиновыми подуровнями.

В $[4,11,12]$ были получены выражения для уменьшения времени $\tau_{T}$ нарастания фосфоресценции в результате опустошения основного состояния. Орнгинальный способ определения спектра триплет-триплетного $(T-T)$ поглощения, основанный на изучении сокращения $\tau_{T}$ в за-

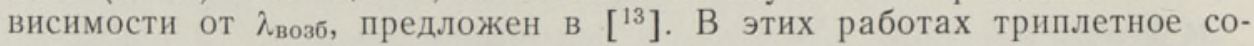
стояние рассматривалось как один уровень, что правомерно лишь в случае быстрой СРР и слабого возбуждения (см. п. 2.3).

В итоге мы пришли к выводу, что процесс заселения нижнего триплетного состояния требует более последовательного учета двух факторов: $T-T$-поглощения и сильного возбуждения. В данной работе мы попытаемся дать компактную трактовку разных сторон этой проблемы, не выходя за рамки приближения линейной оптики и считая заселенности высших возбужденных электронных состояний пренебрежимо малыми. Рассмотрим изолированные молекулы, т. е. не будем принимать во внимание миграцию энергии.

Везде, где это возможно, постараемся получить наглядные, физически интерпретируемые аналитические выражения, а в ряде случаев проиллюстрируем ситуацию результатами численного расчета на ЭВМ. Математически все рассматриваемые задачи сводятся к решению системы линейных дифференциальных уравнений первого порядка с постоянными коэффициентами. Как известно, решения таких систем представляют собой суммы экспонент, причем показатели экспонент являются решениями характеристического алгебраического уравнения.

\section{2. Результаты для разных моделей}

2.1. Влияние $T-T-$ поглоще ния. Рассмотрим четырехуровневую систему, изображенную на рис. $1, a$, отражающую процессы, которые происходят в люминесцирующей молекуле в конденсированной среде. Триплетные состояния $T_{1}$ и $T_{2}$ будем трактовать пока как одиночные уровни. Эта схема предполагает быструю колебательную релаксацию во всех электронных состояниях и не учитывает т. н. горячую передачу энергии между состояниями (см. [14]). Рассмотрим совокупность изолированных идентичных молекул, т. е. однородную примесную систему в тонком слое.

Везде в данной работе интенсивность возбуждения $I_{e x}$ будем считать постоянной во времени, что соответствует возбуждению прямоугольными импульсами. Переходы $S_{1} \leftarrow S_{0}$ и $T_{2} \leftarrow T_{1}$ могут возбуждаться также разными источниками, так как в формулы входят лишь произведения $I_{e x} B_{S} \equiv J_{S}$ и $I_{e x} B_{T} \equiv J_{T}$.

Приступим к решению кинетических уравнений. C учетом сохранения общего числа молекул $N=n_{0}+n_{1}+n_{2}+n_{3}$ для схемы на рис. $1, a$ получим систему из трех дифференциальных уравнений. Однако введение дополнительных упрощающих предположений позволяет уменьшить число зависимых уравнений. Перечислим приближения, которые ниже будут использованы:

а) заселенность $T_{2}$-состояния пренебрежимо мала $-J_{T} \ll R_{3}=$ $=r_{30}+r_{31}+r_{32}$

б) уровень $T_{1}$ затухает медленнее, чем $S_{1}$ и $T_{2}$, а $T_{2}$ быстрее, чем $S_{1}-w_{20} \ll R_{1}=w_{10}+r_{12}, R_{1} \ll R_{3} ;$

в) заселенность $S_{1}$-состояния пренебрежимо мала $-J_{S}, J_{T} \ll R_{1}$.

В приближении (a) уровень $T_{2}$ можно учесть просто в качестве 


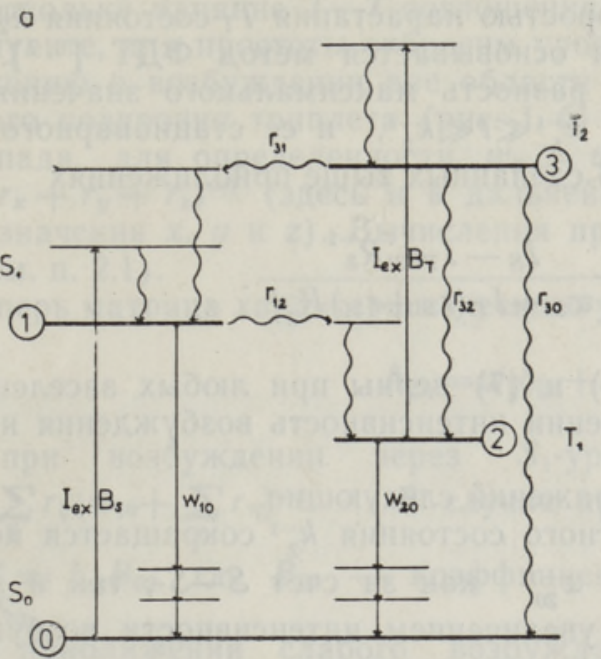

Рис. $1, a$. Типичная схема нижних электронных состояний примесной молекулы и переходов между ними. Волнистые линии означают релаксационные переходы, вероятности распада $w_{10} \cdot$ и $w_{20}$ включают как радиационную, так и безызлучательную части. $I_{e x}-$ интенсивность возбуждения, $B_{S}$ и $B_{T}-$ коэффициенты Әйнштейна для поглощения. б. Три подуровня триплета $T_{1}$. Переходы $\varrho_{x y}$ и $\varrho_{y z}$ - примеры возможных путей СРР.

промежуточного состояния, исключая, таким образом, третье уравнение. Тогда неизвестными останутся заселенности $n_{1} \equiv n_{S}$ и $n_{2} \equiv n_{T}$.

Выпишем выражения для стационарных заселенностей состояний $S_{1}$ и $T_{1}$ в приближениях (а) - (в) :

$$
\begin{gathered}
\tilde{n}_{S}=N \frac{J_{S}}{R_{1}}\left(1+\frac{J_{T}}{w_{20}} \cdot \frac{r_{30}+r_{31}}{R_{3}}\right) D^{-1}, \\
\tilde{n}_{T}=N \varphi_{T} \frac{J_{S}}{w_{20}} D^{-1},
\end{gathered}
$$

где

$$
D=1+\varphi_{T} \frac{J_{S}}{w_{20}}+\frac{J_{T}}{w_{20}} \cdot \frac{r_{30}+\varphi_{S} r_{31}}{R_{3}} .
$$

Здесь $\varphi_{T}=r_{12} / R_{1}-$ т. н. выход $T_{1}$-состояния $\left[{ }^{1}\right], \varphi_{\mathrm{S}}=w_{10} / R_{1}-$ выход флуоресценции в отсутствие тушения $\left(\varphi_{T}+\varphi_{S}=1\right)$. жения

В приближениях (а)-(в) получим для временных констант выра-

$$
\begin{gathered}
k_{1}=R_{1}+\varphi_{S} J_{S}+\varphi_{T} J_{T} r_{31} R_{3}^{-1}+0\left(J_{S}^{2} R_{1}^{-2}\right), \\
k_{2}=w_{20} \cdot D .
\end{gathered}
$$

Временная зависимость заселенности $S_{1}$-состояния состоит из двух компонентов

$$
n_{\mathrm{S}}(t)=\tilde{n}_{\mathrm{S}}-C_{11} \mathrm{e}^{-k_{1} t}+C_{12} \mathrm{e}^{-k_{2} t},
$$


причем второй убывает со скоростью нарастания $T_{1}$-состояния $k_{2} * *$. На изучении этого компонента и основывается метод ФДТ $\left[{ }^{6,7}\right]$. Величину эффекта характеризует разность максимального значения $n_{s}(t)$, наблюдаемого при временах $k_{1}^{-1} \ll t \ll k_{2}^{-1}$, и ее стационарного значения $\Delta n_{\mathrm{S}} \equiv n_{\mathrm{S}}^{\max }-\tilde{n}_{\mathrm{S}}=C_{12}$. В сделанных выше приблнжениях

$$
\frac{\Delta n_{S}}{\tilde{n}_{S}}=\varphi_{T} \cdot \frac{J_{S}-J_{T} r_{31} R_{3}^{-1}}{w_{20}+J_{T}\left(r_{30}+r_{31}\right) R_{3}^{-1}} .
$$

Отметим, что формулы (5) и (7) верны при любых заселенностях $T_{1}$-состояния - в этом отношении интенсивность возбуждения не ограничена.

Выводы из полученных выражений следующие.

Время нарастания триплетного состояния $k_{2}^{-1}$ сокращается по сравнению с временем затухания $w_{20}^{-1}$ как за счет $S-S$-, так и за счет $T-T$-поглощения. Рост $k_{2}$ с увеличением интенсивности возбуждения $I_{e x}$ является линейным. ***

Формула (7) описывает насыщение отношения $\Delta n_{s} / \widetilde{n}_{s}$ при увеличении $I_{e x}$, связанное с тем, что за счет обратных переходов с $T_{1}$ через $T_{2}$ на синглетную систему относительная заселенность $T_{1}$-состояния $n_{T} / n_{0}$ имеет конечный предел. Что касается пути $r_{31}$, то имеются сведения $\left[{ }^{15}\right]$, что в ароматических молекулах такой процесс маловероятен $-r_{31} / R_{3} \sim 10^{-7}$. Надо подчеркнуть, что значение $\Delta n_{s} / \tilde{n}_{s}$ является (если $r_{31}$ мала) прямой мерой относительной заселенности триплета $\tilde{n}_{T} / \tilde{n}_{0}$. Следовательно, ее можно определить непосредственно из кривых убывания флуоресценции, применяя метод ФДТ. Для получения той же информации из кривых нарастания фосфоресценции требуется знать абсолютную величину плотности возбуждения.

Наличие канала $r_{30}+r_{31}$ в наиболее явном виде проявляется в насыщении отношения $\Delta n_{s} / \tilde{n}_{s}$ (см. (7)); интенсивность стационарной фосфоресценции, являясь пропорциональной $\tilde{n}_{T}$ (см. (2) и (3)), насыщается и без $T-T$-возбуждения.

Формула (7) в принципе допускает даже отрицательные значения $\Delta n_{s}$. Это не ошибка - если найдется система, где $J_{T} r_{31}>J_{s} R_{3}$, то в ней кривые $n_{s}(t)$ будут не убывающими, а возрастающими. Независимо от реализуемости такой исключительной ситуации, в случае $r_{31} \neq 0$ заселенность $S_{1}$-уровня не является строго пропорциональной заселенности основного $\left(S_{0}\right)$ уровня, как обычно предполагается $\left[{ }^{3}\right]$ $\left(n_{0}\right.$ легко вычисляется из соотношения $\left.N \simeq n_{0}+n_{T}+n_{S}\right)$.

Для данного типа молекул все полосы или линии люминесценции, различающиеся только по конечным состояниям излучательного перехода, должны иметь одинаковые временные зависимости. Различие кривых $n_{S}(t)$ свидетельствует о неоднородной структуре спектров.

2.2. Три подуровня триплетного состояния. При достаточно низкой температуре, когда СРP замедляется, необходимо учитывать расщепление триплетного состояния на три подуровня. В данном пункте рассмотрим случай, когда СРР полностью отсутствует.

** Мы можем по-прежнему называть $k_{2}$ скоростью нарастания $T_{1}$, так как вклад компонента $\exp \left(-k_{1} t\right)$ в заселенность $n_{T}$ мал - порядка $w_{20} R_{1}^{-1}$.

*** При $I_{e x} \rightarrow \infty$ (допускаются значения $J_{s} \geqslant R_{1}$ ) скорость $\dot{k}_{2}$ имеет асимптотику вида $k_{2}^{\text {ac }}=r_{12}\left(B_{S} R_{3}-B_{T} r_{31}\right)\left(B_{S} R_{3}-B_{T}\left(r_{30}+r_{31}\right)\right)^{-1}+J_{T}\left(r_{30}+r_{31}\right) R_{3}^{-1}$. 
Поскольку влияние $T-T$-поглощения было рассмотрено в предыдущем пункте, для простоты отбросим уровень $T_{2}$, что эквивалентно предположению о возбуждении вне области $T-T$-поглощения. Введем для каждого подуровня триплета (рис. $1, \sigma)$ вероятности заселения $r_{x}, r_{y}, r_{z}$ и распада, для определенности $w_{x}>w_{y}>w_{z}$, и обозначим $p_{\xi}=$ $=r_{\xi}\left(r_{x}+r_{y}+r_{z}\right)^{-1}$ (здесь и в дальнейшем греческие индексы пробегают значения $x, y$ и $z$ ). Вычисления проводим в приближениях (б) и (в) (см. п. 2.1) .

Теперь матрица характеристического уравнения имеет вид

$$
\mathbf{A}_{\xi \eta}=w_{\xi} \delta_{\xi \eta}+J p_{\xi},
$$

где при возбуждении через $S_{1}$-уровень $J=I_{e x} B_{S} \varphi_{T}, \quad$ причем $\varphi_{T}=\sum_{\xi} r_{\xi}\left(w_{10}+\sum_{\eta} r_{\eta}\right)^{-1}$. В случае прямого возбуждения $T_{1}$-состояния $J=I_{e x} B_{02}$, где $B_{02}-$ коэффициент Эйнштейна для перехода $T_{1} \leftarrow S_{0}$.

В приближении слабого возбуждения, $J \ll w_{\text {ह }}$ корнями этого уравнения являются $k_{\xi}=w_{\xi}+J p_{\xi}$. Очевидно, в линейном приближении по $J$ для каждого подуровня отдельно применимы результаты п. 2.1 - заселение происходит независимо.

В пределе сильного возбуждения, $J \gg w_{\text {, }}$, один из корней $k_{x}^{\mathrm{ac}}=J\left(p_{x}+p_{y}+p_{z}\right)=J$ неограниченно растет, а два остальных приближаются к конечным значениям. Изменение скоростей $k_{\xi}$ с ростом $J$ проиллюстрируем на примере рис. 2. Здесь принята модель, в которой времена распада всех подуровней хорошо различимы. В модели $A$ вероятности заселения $p_{\xi}$ и распада $w_{\xi}$ попарно пропорциональны - этот случай приближенно реализуется для многих молекул $[9,10]$. Для сравнения рассмотрена модель $Б$, где заселение не является избирательным, этот случай также встречается в практике $\left[{ }^{16}\right]$. Из рис. 2 видно, что в варианте $A$ рост двух медленных компонентов $k_{y}$ и $k_{z}$ незначителен, а в варианте 5 заметен явный скачок.

Рис. 2. Зависимость от приведенной интенсивности возбуждения $J$ коэффициентов затухания экспонент $\exp \left(-k_{\xi} t\right)$ в кинетике заселения трех подуровней $(x, y$, z) триплета при отсутствии СРP. Здесь и на следующих рисунках сплошная линия соответствует модели $A: \quad w_{x}=80, \quad w_{y}=16$, $w_{z}=4 \quad$ и $\quad p_{x}=0,8, \quad p_{y}=0,16$, $p_{z}=0,04$, штриховая линия модели $Б$ : $w_{\xi}$ те же, $p_{x}=p_{y}=$ $=p_{z}=1 / 3 \quad$ (параметры $k_{\xi}, w_{\xi}$ измеряются в произвольных единицах). Приведены отношения $k_{\xi}$ К их начальным значениям

$$
k_{\S}^{0}=w_{\S} \text { при } J=0 .
$$

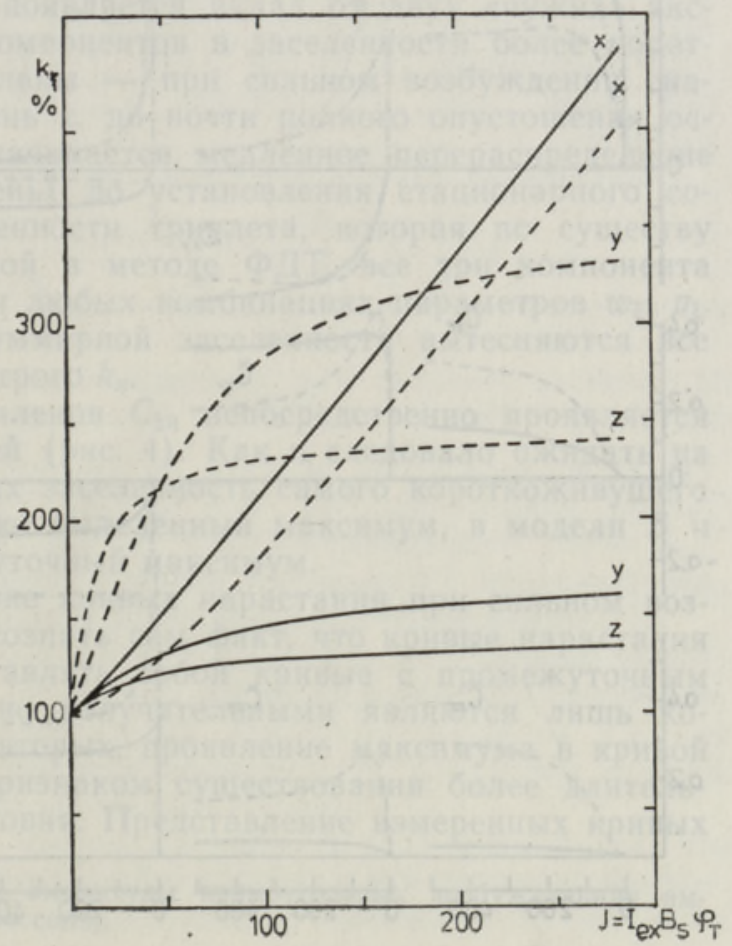


Конечность $k_{y}$ и $k_{z}$ все же соблюдается только без учета $T-T$-поглощения - нетрудно убедиться, что аналогично формуле (5) при включении $T-T$-переходов во всех $k_{\xi}$ появляются члены, пропорциональные $J_{T}$. С целью упрощения кинетики возбуждение следует выбирать вне области $T-T$-поглощения (часто это возможно).

Выражение для стационарных заселенностей аналогично выражению для одного подуровня (2):

$$
\tilde{n}_{\xi}=N J \frac{p_{\xi}}{w_{\xi}}\left(1+J \sum_{\eta} \frac{p_{\eta}}{w_{\eta}}\right)^{-1} .
$$

Отсюда видно, что отношения стационарных заселенностей подуровней в данной модели не зависят от интенсивности возбуждения (и этот результат получается за счет отсутствия $T-T$-поглощения). В варианте $p_{\xi} / w_{\xi}=$ const стационарные заселенности всех подуровней одинаковы, а в случае $p_{\xi}=$ const на более долгоживущих уровнях накапливается больше молекул.

Не менее важными кинетическими параметрами являются предэкспоненциальные множители в решениях системы

$$
n_{\xi}(t)=\tilde{n}_{\xi}-\sum_{\eta} C_{\xi \eta} \mathrm{e}^{-k} \eta^{t} .
$$

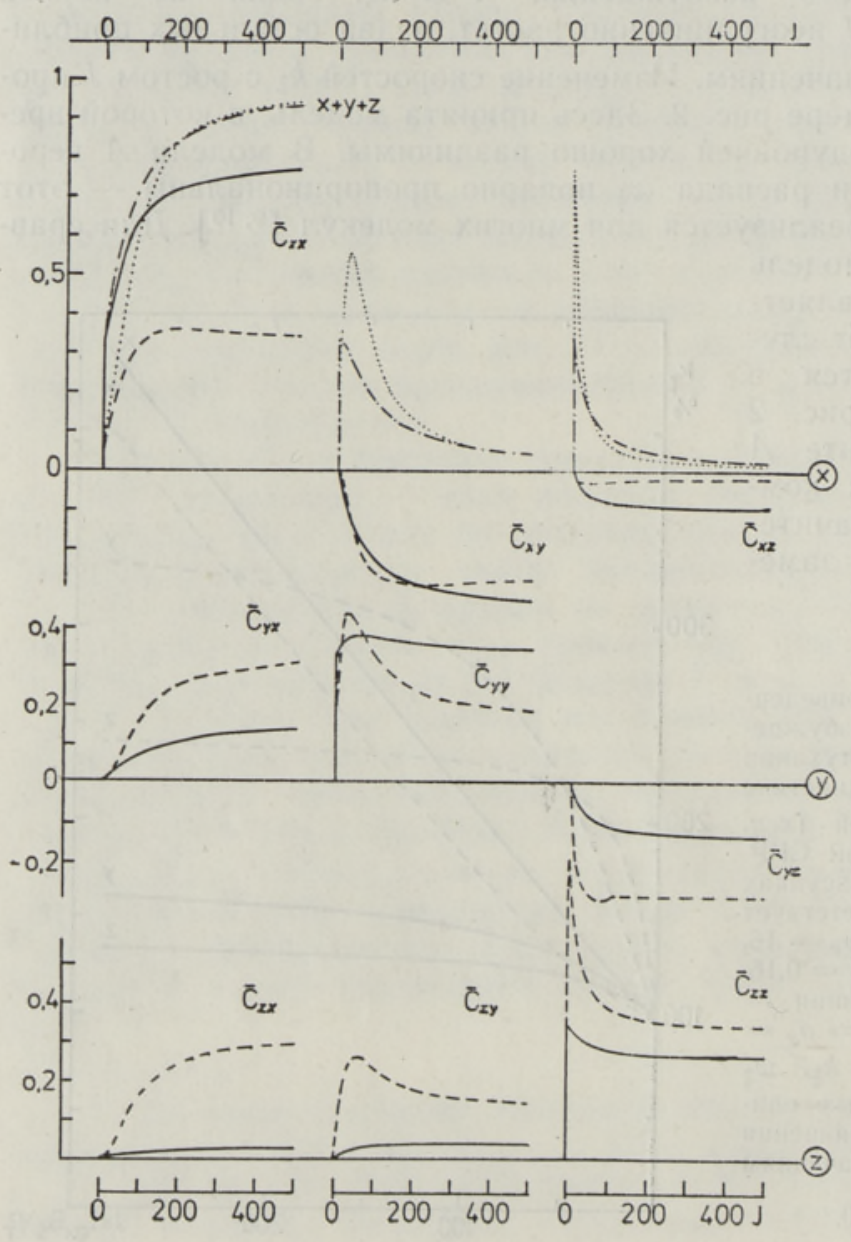

Рис. 3. Зависимость от интенсивности возбуждения предэкспоненциальных множителей $\bar{C}_{\xi \eta}=C_{\xi \eta} / \Sigma C_{\xi \eta}$ для моделей $A$ и $Б$. Кривые $x+y+z$ представляют собой соответствующие коэффициенты в суммарной заселенности, т. е. суммы $\Sigma \bar{C}_{\text {छп }}$ для $A$ s (-...- ) и и $Б$ Б (....). 
Рис. 4. Временная зависимость относительных заселенностей отдельных подуровней триплета $(\varrho=0)$. При $t=0$ включается возбуждение с интенсивностью $J=100$, начальные условия $n_{\xi}=0$.

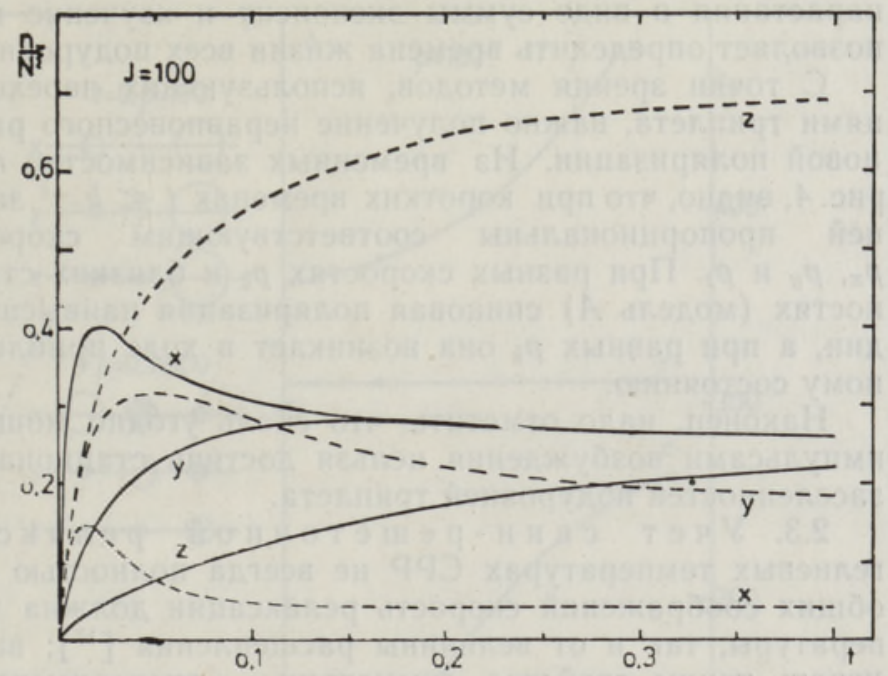

На рис. 3 изображена зависимость элементов матрицы $C_{\xi \eta}$ от интенсивности возбуждения при нулевых начальных условиях $n_{\text {૬ }}(0)=0$. Здесь наглядно проявляется эффект смешивания кинетик заселения отдельных подуровней при увеличении интенсивности возбуждения. В этом смысле в ходе возбуждения как бы происходит релаксация между спиновыми подуровнями через основное состояние. Все же аналогия лишь частична, так как включение $J$ не эквивалентно какой-либо комбинации СРP (ср. п. 2.3) .

Сущность результатов, приведенных на рис. 3, заключается в следующем. При возраста̇нии интенсивности возбуждения **** в заселенностях $n_{\xi}$ всех трех уровней появляется вклад от двух «чужих» экспонент. Вклады медленных компонентов в заселенности более короткоживущих уровней отрицательны - при сильном возбуждении сначала быстро заселяется уровень $x$, до почти полного опустошения основного состояния, а затем начинается медленное перераспределение заселенностей (через $S_{0}$-уровень) до установления стационарного состояния. В суммарной заселенности триплета, которая по существу является измеряемой величиной в методе ФДТ, все три жомпонента остаются положительными при любых комбинациях параметров $w_{\xi}, p_{\xi}$. Как видно, при $J \rightarrow \infty$ из суммарной заселенности вытесняются все компоненты, кроме самого быстрого $k_{x}$.

Влияние недиагональных членов $C_{\xi \eta}$ непосредственно проявляется в кривых заселения подуровней (рис. 4). Как и следовало ожидать на основе рис. 3 , в обеих моделях заселенность самого короткоживущего уровня $n_{x}$ проходит через четко выделенный максимум, в модели $E$ и заселенность $n_{y}$ имеет промежуточный максимум.

Что же может дать изучение кривых нарастания при сильном возбуждении? Во-первых, надо осознать сам факт, что кривые нарастания фосфоресценции могут представлять собой кривые с промежуточным максимумом, поскольку обычно излучательными являются лишь короткоживущие подуровни. Во-вторых, проявление максимума в кривой нарастания может служить признаком существования более длительного нефосфоресцирующего уровня. Представление измеренных кривых

**** Имеется в виду изменение амплитуды прямоугольных возбуждающих импульсов, а за период возбуждения $J=$ const. 
нарастания в виде суммы экспонент и изучение их зависимости от $J$ позволяет определить времена жизни всех подуровней.

С точки зрения методов, использующих переходы между подуровнями триплета, важно получение неравновесного распределения - спиновой поляризации. Из временных зависимостей $n_{\xi}(t)$, показанных на рис. 4 , видно, что при коротких временах $t \ll k_{x}^{-1}$ заселенности подуровней пропорциональны соответствующим скоростям возбуждения $p_{x}, p_{y}$ и $p_{z}$. При разных скоростях $p_{\xi}$ и близких стационарных заселенностях (модель $A$ ) спиновая поляризация наивысшая в начальной стадии, а при равных $p_{\xi}$ она возникает в ходе приближения к стационарному состоянию.

Наконец, надо отметить, что сколь угодно мощными, но короткими импульсами возбуждения нельзя достичь стационарных относительных заселенностей подуровней триплета.

2.3. Учет спин-решеточной релаксация. Даже при гелиевых температурах СРP не всегда полностью замораживается. Из общих соображений скорость релаксации должна зависеть как от температуры, так и от величины расщепления $\left[{ }^{17}\right]$; важную роль должны играть также свойства симметрии - экспериментально наблюдались $\left[{ }^{18,19}\right]$ разные соотношения скоростей, не имеющие однозначной корреляции с энергией переходов.

Выпишем матрищу А (ср. (8)) для общего случая релаксирующей системы с тремя подуровнями:

$$
\mathbf{A}=\left(\begin{array}{ccc}
w_{x}+J p_{x}+\varrho_{x y}+\varrho_{x z} & J p_{x}-\varrho_{y x} & J p_{x}-\varrho_{z x} \\
J p_{y}-\varrho_{x y} & w_{y}+J p_{y}+\varrho_{y x}+\varrho_{y z} & J p_{y}-\varrho_{z y} \\
J p_{z}-\varrho_{x z} & J p_{z}-\varrho_{y z} & w_{z}+J p_{z}+\varrho_{z x}+\varrho_{z y}
\end{array}\right),
$$

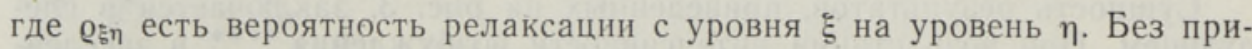
ложения внешнего высокочастотного поля пары переходов @п $=$ $=\varrho_{\xi \eta} q_{\xi \eta}$ в силу принципа детального равновесия связаны через факторы Больцмана $q_{\xi \eta}=\exp \left[\left(E_{\eta}-E_{\xi}\right) / k T\right]$. Для расщеплений в нулевом поле даже температура $1 \mathrm{~K}$ столь высока, что практически $\mathrm{Q}_{\eta}=\mathrm{Q \Sigma}_{\eta}$.

Выражение для стационарных заселенностей имеет вид

$$
\begin{gathered}
\tilde{n}_{x}=N \frac{J}{w_{x}}\left[p_{x}\left(1+\frac{\varrho_{y x}+\varrho_{y z}}{w_{y}}+\frac{\varrho_{z x}+\varrho_{z y}}{w_{z}}\right)+p_{y} \frac{\varrho_{y x}}{w_{y}}+p_{z} \frac{\varrho_{z x}}{w_{z}}+\right. \\
\left.+\frac{1}{w_{y} w_{z}} \cdot\left(\varrho_{y x} \varrho_{z x}+\varrho_{y z} \varrho_{z x}+\varrho_{z y} \varrho_{y x}\right)\right] D^{-1},
\end{gathered}
$$

где $D$ не зависит от индекса подуровня. Формулы для $\tilde{n}_{y}$ и $\tilde{n}_{z}$ совершенно аналогичны. Из (12) следует, что при быстрой релаксации $\left(\varrho_{\bar{n}} \rightarrow \infty\right)$ заселенности будут различаться лишь за счет температурных факторов, а при $\varrho_{\ddagger} \rightarrow 0$ получим результат формулы (9).

При одновременном увеличении всех @६п скорости $k_{\xi}$, строго говорн, будут зависеть от соотношений между компонентами @६ฑ. Поскольку

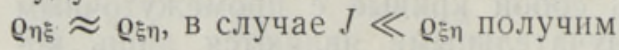

$$
k_{z}^{\mathrm{ac}}=\frac{1}{3} \sum_{\xi} w_{\xi}+J \sum_{\xi} p_{\xi}
$$

что по существу представляет собой константу нарастания для системы с одним возбужденным уровнем (ср. с формулой (5)). В то же время 


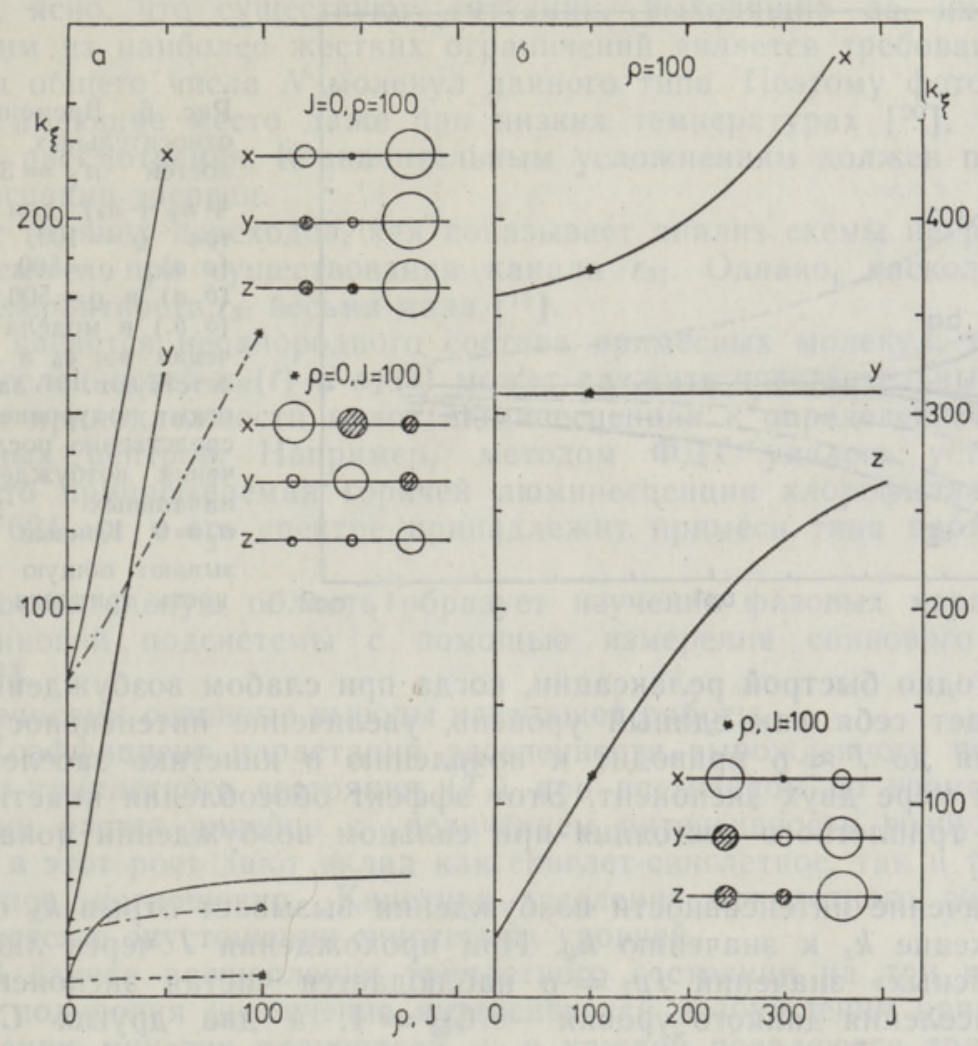

Рис. 5 , а. Зависимость показателей экспонент $k_{\xi}$ от скорости СРР @ при $J=0$ (сплошная линия) для модели $A$. Штрих-пунктирной линией показана зависимость $k_{\xi}$ от $J$ при $\varrho=0$. Справа изображена структура матрицы коэффициентов $C_{\xi \eta}$ для двух комбинаций $J$ и $\varrho$, причем величину компонентов $C_{\xi \eta}$ характеризует площадь кружков (заштрихованные кружки соответствуют отрицательным значениям). б. Зависимость $k_{\xi}$ от $J$ при $Q=100$ (модель $A$ ) и матрица $C_{\xi \eta}$ при $Q=100, J=100$.

$k_{x}$ и $k_{y}$ растут линейно по @эп - они переходят в чисто релаксационные члены.

В дальнейшем для наглядности примем все скорости релаксации равными: $\varrho_{\ddagger}=\varrho$. Это, по-видимому, достаточно общий случай для высокотемпературной области.

Тогда при $\varrho \rightarrow \infty \quad k_{z}$ по-прежнему определяется по (13) и $k_{x}^{\mathrm{ac}}=k_{y}^{\mathrm{ac}}=3 \mathrm{Q}, \quad$ но при равных начальных условиях $n_{x}(0)=n_{y}(0)=$ $=n_{z}(0)$ вся кинетика происходит со скоростью $k_{z}$. Если удается нарушить равновесие, то релаксация происходит со скоростью $3 \varrho$.

Зависимость констант затухания от е иллюстрирована на примере модели $A-$ рис. 5, a. Для сравнения воспроизведена также зависимость $k_{\xi}$ от $J$ при $\varrho=0$ и изображена структура матрицы $C_{\xi \eta}$ для $\varrho=\Sigma w_{\xi}$ и $J=\Sigma w_{\xi}$. Здесь наглядно проявляется различное влияние параметров е и $J$ на кинетику триплетного состояния.

Уже вид недиагональных членов матрицы А (11) показывает, что при интенсивностях $J \approx \varrho$ происходит своеобразная взаимная компенсация $J$ и $е$. Подробный анализ показывает, что даже в условиях 


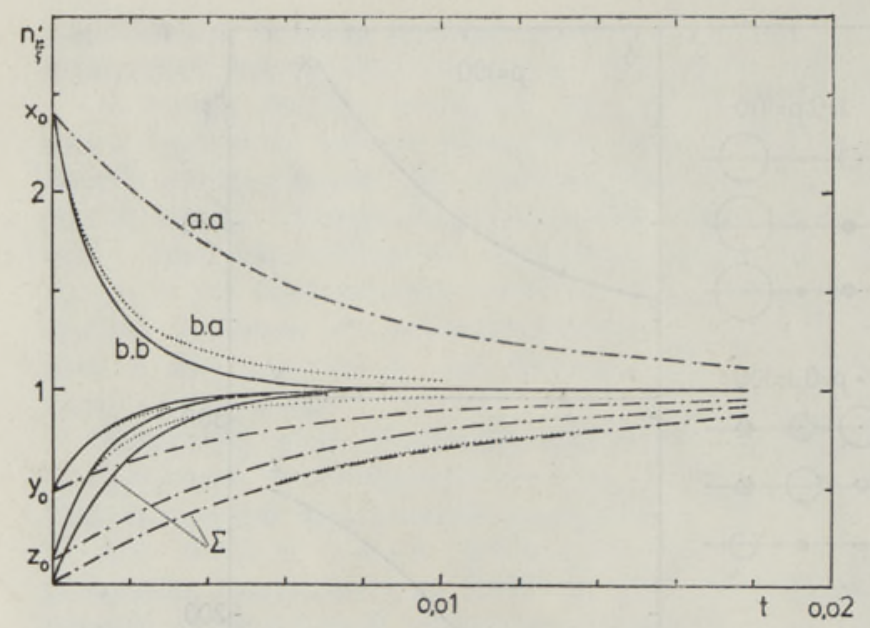

Рис. 6. Временной ход относительных заселенностей $n_{\xi}^{\prime}=3 n_{\xi} /\left(n_{x}+\right.$ $+n_{y}+n_{z}$ ) для вариантов $\quad g=100, \quad J=100$ (a. a), $\varrho=500, \quad J=100$ (b. a) и $\mathrm{Q}=500, \quad J=500$ (b.b.) в модели $A$. Значения $x_{0}, y_{0}$ и $z_{0}$ соответствуют заселенностям подуровней непосредственно после включения возбуждения при начальных условиях $n_{\xi}=0$. Кривые $\Sigma$ показывают общую заселенность триплета $\Sigma n_{\xi} / N$.

сколь угодно быстрой релаксации, когда при слабом возбуждении триплет ведет себя как единый уровень, увеличение интенсивности возбуждения до $J \approx$ q приводит к появлению в кинетике заселения по меньшей мере двух экспонент. Этот эффект обособления кинетик подуровней триплетного состояния при сильном возбуждении показан на рис. 5, б.

Увеличение интенсивности возбуждения вызывает отрыв $k_{x}$ от $k_{y}$ и приближение $k_{z}$ к значению $k_{y}$. При прохождении $J$ через любое из «резонансных» значений $J p_{\xi}=\varrho$ наблюдается чистая экспоненциальность заселения данного уровня $-C_{\xi \xi}=1$, а два других $C_{\xi \eta}=0$, т. е. уровень $\xi$ заселяется только со «своей» константой нарастания $k_{\xi}=w_{\xi}+3 \varrho$.

Различия в кинетике заселения отдельных подуровней приводят к наведению спиновой поляризации даже в быстрорелаксирующей системе, где $\tilde{n}_{x} \approx \tilde{n}_{y} \approx \tilde{n}_{z}$. Это обусловлено тем, что при наличии релаксации заселенности в начальной стадии по-прежнему пропорциональны вероятностям заселения $p_{\xi}$. Однако в данном случае это верно лишь для времен, малых и по отношению к релаксации $\left(t \ll Q^{-1}\right)$, так что при слабом возбуждении за это время триплетное состояние просто не успевает сколь-нибудь значительно заселиться (см. рис. 6). Видно, что в области $t<(3 \varrho)^{-1}$ имеется значительная спиновая поляризация, причем доведение интенсивности возбуждения до $J=\varrho$ позволяет достичь за это время общей заселенности триплетного состояния до $30 \%$. Для той же модели $A$ возникающая при распаде начального состояния $n_{x}=n_{y}=n_{z}$ спиновая поляризация достигает при $\varrho=100$ предельного значения $\left(n_{z}-n_{x}\right)\left(n_{z}+n_{x}\right)^{-1} \simeq 12 \%$, а при $\varrho=500$ всего лишь $2,5 \%$.

Итак, приходим к заключению, что при разных вероятностях заселения подуровней временную спиновую поляризацию можно навести короткими мощными импульсами фотовозбуждения. При равновероятном заселении всех подуровней спиновая поляризация возникает лишь в случае медленной релаксации за счет разных скоростей распгда.

\section{3. Обсуждение и выводы}

В первую очередь следует обсудить пределы применимости результатов данной работы. Хотя мы старались рассмотреть довольно общие 
модели, ясно, что существуют ситуации, выходящие за их рамки.

Одним из наиболее жестких ограннчений является требование сохранения общего числа $N$ молекул данного типа. Поэтому фотопревращения, имеющие место даже при низких температурах [ $\left.{ }^{20}\right]$, требуют особого рассмотрения. К значительным усложнениям должен привести учет миграции энергии.

Учет горячих переходов, как показывает анализ схемы на рис. $1, a$, имеет смысл при существовании канала $r_{31}$. Однако, насколько известно, вероятность $r_{31}$ весьма мала $\left[{ }^{15}\right]$.

Что касается неоднородного состава примесных молекул, то кинетика заселенностей $n_{S}(t)$ и $n_{T}(t)$ может служить чувствительным индикатором принадлежности полос люминесценции к определенному типу примесных центров. Например, методом ФДТ удалось установить $\left[{ }^{6,7}\right]$, что приписываемая горячей люминесценции хлорофилла $a\left[{ }^{21}\right]$ полоса 624 нм в его спектре принадлежит примеси типа протохлорофилла.

Самостоятельную область образует изучение фазовых характеристик спиновой подсистемы с помощью измерения спинового эха и т. п. $\left[{ }^{22}\right]$.

Перечислим основные выводы настоящей работы.

1. Коэффициент нарастания заселенности вырожденного по спину нижнего триплетного состояния $\left(T_{1}\right)$ при постоянном во времени возбуждении растет линейно с увеличением интенсивности возбуждения, причем в этот рост дают вклад как сннглет-синглетное, так и триплеттриплетное поглощения. Кинетика заселения триплетного состояния проявляется в опустошении синглетных уровней.

2. В случае расщепления триплетного состояния на три изолированных подуровня увеличение интенсивности возбуждения приводит к смешиванию кинетик подуровней - в каждой появляются три экспоненты. Наивысшая из скоростей заселения растет линейно, а остальные в отсутствие $T-T$-поглощения приближаются к конечным значениям.

3. При сильном возбуждении в ходе приближения к стационарному состоянию заселенность самого короткоживущего подуровня проходит через промежуточный максимум.

4. При наличии СРР два показателя экспонент растут линейно по параметру СРP @ и представляют собой релаксационные члены, лишь наименьший из показателей превращается в усредненную скорость заселения триплета.

5. При определенных значениях интенсивности возбуждения $J$ происходит «интерференция» $J$ и $\mathbf{Q}$, в результате чего кинетика заселения одного из подуровней становится чисто экспоненциальной. Как и в отсутствие СРР, короткими импульсами можно создавать неравновесное распределение между подуровнями, т. е. наводить спиновую поляризацию.

Выражаю благодарность К. Ребане за полезные замечания.

\section{Л И Т Е Р А У Р А}

1. Мак-Глинн С., Адз уми Т., Киносита М., Молекулярная спектроскопия триплетного состояния, М., 1972.

2. Dut to n, P. L., Le ig h, J. S., S i e bert, M., Biochem. and Biophys. Res. Communs, $46,406(1972)$.

3. E 1-S a y e d, M. A., In: Excited States, ed. C. Lim, 1, New York a. London, 1974, p. 35 .

4. С ми рнов В. А., Ал фимов М. В., Кинетика и катализ, 7, 583 (1966). 
5. Van Dorp, V. G., S choemaker, W. H., S om a, M., Van der W a al s, J. H., Molec. Phys., 30, 1701 (1975).

6. А в а р м а а Р., М а ур инг К., Изв. АН ЭССР, Физ. Матем., 26, 92 (1977).

7. A va rm a a, R., Chem. Phys. Letters, 46, 279 (1977).

8. С в еш н и ков Б. Я., ЖЭТФ, 18,878 (1948).

9. S c hwoere r, M., S ix l, H., Z. Naturforsch., 24a, 952 (1969).

10. Kleibeuker, J. F., S cha a f sma, T. J., Chem. Phys. Letters, 29, 116 (1974).

11. K in os hita, M., Srin ivasan, B. N., Mc Glynn, S. P., J. Molec. Spectr., 21, 335 (1966).

12. N a ka mizo, M., Matsueda, T., J. Molec. Spectr., 27, 450 (1968).

13. Болотникова Т. Н., Н аумова Т. М., Коротаева Е. А., Тимофсе в а Ю. Ф., Изв. АН СССР, Сер. физ., 39, 2331 (1975).

14. Ребане К., Вторичное свечение примесного центра кристалла, Тарту, 1970; Хи жняков В. В., Техвер И. Ю., Изв. АН СССР, Сер. физ., 39, 1895 (1975).

15. Keller, R. A., In: Molecular Luminescence, ed. E. C. Lim, New York a. Amsterdam, 1969, p. 453.

16. D i n se, K. P., M a ki, A. H., Chem. Phys. Letters, 38, 125 (1976).

17. А ле кс анд ро в И. В., Теория магнитной релаксации, М., 1975.

18. H a 11, L. H., E 1-S a y e d, M. A., J. Chem. Phys., 54, 4958 (1971).

19. Z u cli ch, J., S chütz, J. U., Maki, A. H., Molec. Phys., 28, 33 (1974).

20. Го роховски й А. А., Опт. и спектр., 40, 477 (1976).

21. Menzel, E. R., Polles, J. S., Chem. Phys. Letters, 24, 545 (1974).

22. Harris, C. B., Schlupp, R. L., Schuch, H., Phys. Rev. Letters, 30, 1019 (1973).
Ннститут физики
Академии наук Эстонской ССР
Поступила в редакцио
$17 / 1 \quad 1977$

\section{R. AVARMAA}

\section{LISANDIMOLEKULI TRIPLETSE SEISUNDI ASUSTAMISKINEETIKA}

Artiklis on tuletatud valemid, mis kirjeldavad tripletse seisundi asustamiskineetikai järgmiste mudelite najal: (1) elektronseisundeid $S_{0}, S_{1}, T_{1}$ ja $T_{2}$ hōlmav neljanivooline skeem; (2) sama skeem, kuid puudub $T_{2}$-nivoo ja on arvestatud seisundi $T_{1}$ kolme alanivood; (3) tripleti alanivoode vahelist relaksatsiooni arvestav mudel. On kăsitletud่ ergastamist nelinurkimpulssidega ülemineku $S_{1} \leftarrow S_{0}$ kaudu. Mudeli (1) abil on järjekindlalt arvesse võetud $T-T$-neeldumise mōju, (2) puhul ilmneb alanivoode kineetikate segunemine. Variandis (3) on leitud relaksatsiooni tōttu seotud alanivoode kineetikate lahknemise efekt ning sellest tulenev ajutine spinnpolarisatsioon, mida saab esile kutsuda tugeva ergastusega.

\section{R. AVARMAA}

\section{TRIPLET STATE POPULATION KINETICS OF THE IMPURITY MOLECULE}

Formulae are derived, describing the triplet state population kinetics within the following models: (1) four-level scheme including the electronic states $S_{0}, S_{1}, T_{1}$ and $T_{2}$; (2) the same scheme without $T_{2}$-level, but taking into account three sublevels of $T_{1}$-state; (3) model including relaxation between the triplet sublevels. The square-wave excitation via $S_{1} \leftarrow S_{0}$ transition is considered. With the use of the model (1) the influence of the $T-T$-absorption is consistently treated; in the case (2) the mixing of the sublevels kinetics is shown to arise. Within the model (3) the effect of decoupling the relaxationally coupled sublevels by intense excitation is found, together with the resulting induced transient spin-polarization. 